\title{
Téoros
}

Revue de recherche en tourisme

\section{Isla Navidad : un méga-projet touristique au Mexique}

\section{José Antonio Murillo}

Volume 13, numéro 2, été 1994

Regards sur le tourisme dans les pays en développement

URI : https://id.erudit.org/iderudit/1077763ar

DOI : https://doi.org/10.7202/1077763ar

Aller au sommaire du numéro

Éditeur(s)

Université du Québec à Montréal

ISSN

0712-8657 (imprimé)

1923-2705 (numérique)

Découvrir la revue

Citer cet article

Murillo, J. A. (1994). Isla Navidad : un méga-projet touristique au Mexique.

Téoros, 13(2), 39-42. https://doi.org/10.7202/1077763ar d'utilisation que vous pouvez consulter en ligne.

https://apropos.erudit.org/fr/usagers/politique-dutilisation/ 


\title{
Isla Navidad : un méga-projet touristique au Mexique
}

\author{
José Antonio Murillo*
}

Le20 mars 1972, le méga-projet Isla Navidad est amorcé avec l'acquisition d'une réserve territoriale de 360 hectares, constituée de domaines connus sous le nom de La Culebra ou lsla de San Francisco, localisés dansla municipalité deManzanillo, Colima; le 20 octobre 1973,20 autres hectares sont alors enregistrés.

Cette superficie permet à Isla Navidad de se comparer, sur le plan touristique à des sites et des centres touristiques réalisés par leGouvernement Fédéral du Mexique-via le Fonds National de Développement du Tourisme(FONATUR) et le ministère du Tourisme - comme Cancún, Ixtapa Zihuatanejo, San José del Cabo, Loreto Napoló et Huatulco-Puerto Escondido.

L'emplacement géographique de l'Isla Navidad, sur la Costa de Oro du Pacifique, caractérisée par une prolifération de pôles touristiques (certains étant consolidés, d'autres en voie de développement et d'autres encore à l'étape initiale ou interrompus), confirme ce que le ministre du Tourisme faisait remarquer en 1987 , à savoir que le projet s'établit dans une région qui «présente un potentiel élevé de développement touristiques.

Le promoteur du projet Isla Navidad - le Groupe Barramar - possède une solide expérience dansla planification, l'exécution et la gestion de projets immobiliers à caractère touristique et résidentiel, ainsi qu'une structure corporative diversifiée dansles secteursdela presse, del'éducation, de l'agro-industrie, des sports, de la construction et de la santé ce qui renforce davantage la faisabilité du projet.

Le méga-projet touristique repose sur une planification urbaine et sur des études techniques garantissant l'approvisionne-

Monsieur José Antonio Murillo est directeurcoordonnateur exécutif du méga-projet touristique Isla Navidad a Manzanillo ment en eau et en énergie électrique, afin de préserver et d'enrichir le milieu naturel ambiant et mettre en évidence la flore de la région; il repose également sur des études demarchés et desévaluations financières et économiques de l'ensemble du projet ainsi que d'autres projets spécifiques.

Le méga-projet Isla Navidad implique l'évaluation de la viabilité d'un projet dont l'envergure équivaut aux deux tiers de celle qui avait été prévue pour Cancún. Cela signifie aussi l'évaluation d'un projet entrepris intégralement par l'initiative privée, avec ses propres ressources, et nécessitant un coût financier réel en comparaison des investissements touristiques gouvernementaux consentis dans le cadre de ressources budgétées, sans coût financier réel. Cela implique également le défi de concevoir un nouveau pôle touristique qui peut se différencier de ceux actuels et qui, en même temps, peut compléter et enrichir l'offre touristique du Mexique et bien se positionner aux niveaux national et international, chez une catégorie de touristes qui se démarquent de plus en plus par un haut niveau de sophistication et qui réclament davantage que le folklore, le soleil et la mer.

\section{Contexte macro-économique de l'industrie touristique}

Contribution de l'industrie touristique à l'économie nationale

De 1970 à 1990 , le produit intérieur brut de l'activité touristique (restaurants et hôtels) enregistrait un taux de croissance annuel moyen de $4,5 \%$. Au cours de ces années, on peut distinguer trois grandes périodes.

Pendant les années 1970, on assiste à une période d'expansion et d'essor pendant laquelle la valeur de l'activité touristique passe de 67,5 milliards de pesos en 1970 à 152,3 milliards de pesos constants en 1980; soit un taux de croissance moyen de $8,5 \%$.

Au cours des années 1980 à 1985 , ce fût une période de récession de l'activité touristi- que où la valeur a diminué de 152,3 à 129,5 milliards de pesos constants, soit un taux négatif de $3,2 \%$.

À partir de 1985, on a constaté une relance de l'activité touristique qui s'est traduite par une augmentation du PIB touristique de 129,5 milliards à 153,5 milliards en 1990; soit une augmentation annuelle de $3,5 \%$. Cette relance paraît s'être consolidée et même renforcée entre 1990 et 1992.

Les perspectives pour les prochaines années, dans le secteur touristique, sont positives et ainsi encourageantes pour de nouveaux investissements qui proviendront, en grande partie, du secteur privé, comme le souhaite la politique gouvernementale qui tend à «laisser plus de place à l'initiative privées. On peut considérer que le climat pour investir, dans la conjoncture actuelle, est plus prometteur et comporte moins de risques que durant les huit premières années de la décennie 1980 .

Àcompter de 1989, un ensembledemesures politiques, économiques et financières, appuyé par des mesures de concertation ont donné une impulsion à la croissance économique par le biais d'investissements privés nationaux et étrangers. Tout ceci, pris dans son ensemble, laisse présager des perspectives positives pour le développement du tourisme.

Les caractéristiques du tourisme mexicain et son influence dans le marché touristique mondial.

L'ampleur du marché touristique mondial s'est accrue de 2,8 fois au cours des 20 dernièresannées, passant de 160 millions de touristes en 1970 , à 444 millions en 1990, soit un taux de croissance moyen de $5,2 \%$.

Au cours de cette période, le Mexique a enregistré une progression moyenne de $1,4 \%$. Évidemment, ce résultat ne correspondait pas au potentiel du pays face aux autres destinations touristiques. 


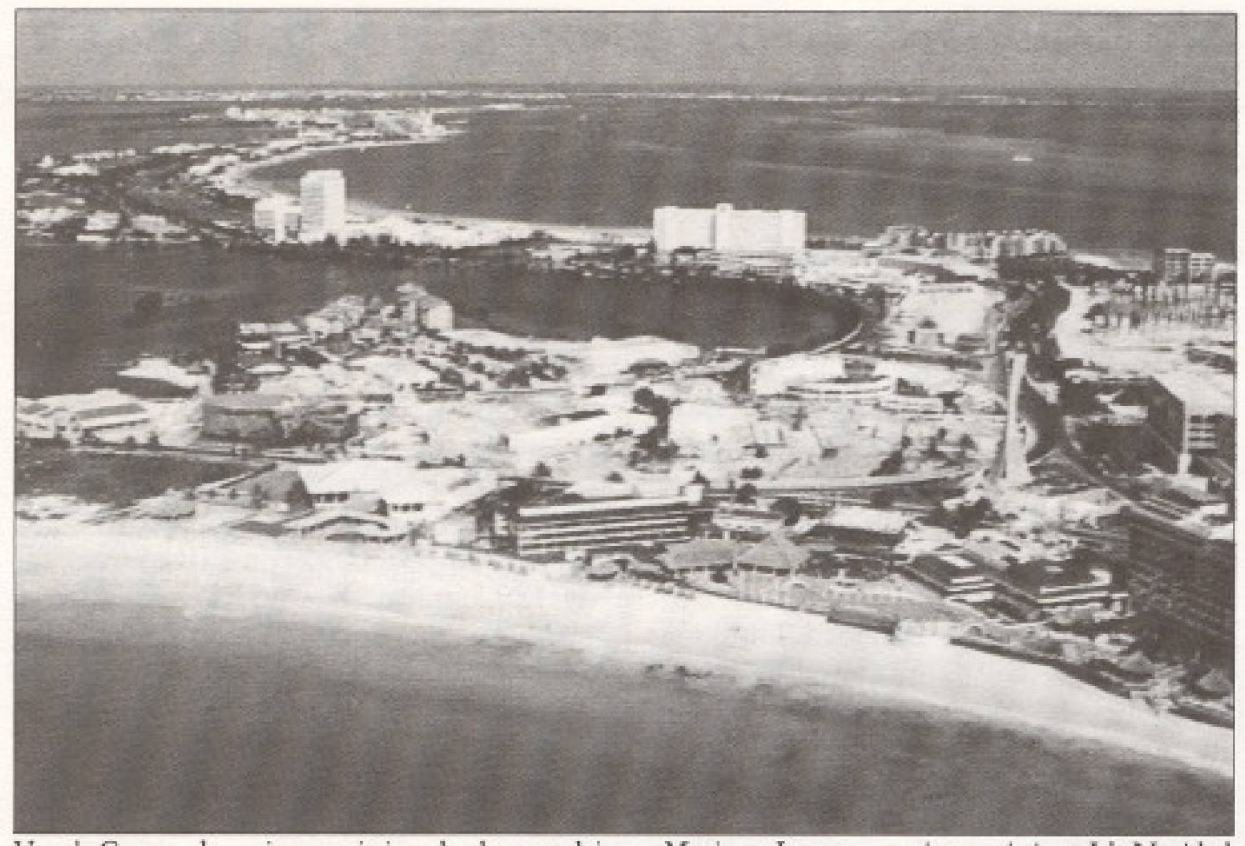

Vue de Cancun, la station touristique la plus populaire au Mexique. Le mega-projet touristique Isla Navidad equivaut aux deux-tiers de Cancun.

Entre 1990 et 1995 , conformément aux perspectives de croissance, on estime que le tourisme réceptif pourtait s'accroitre d'un taux moyen variant entre $7,4 \%$ et $9,4 \%$. Ces chiffres sont des indicateurs qui témoignent des perspectives considérables du développement du tourisme dans le pays.

\section{Isla Navidad et son environnement socio-économique et urbano- touristique}

\section{La localisation géographique}

Le terrain où se développe le méga-projet Isla Navidad est localisé dans la municipalité deManzanillo, Cerro de San Francisco ou Cerro de la Culebra, dans l'état de Colima. Il est situé en face de la localité de Barra de Navidad, dans la municipalité de Cihuatlắn, état de Jalisco.

La localisation privilégiée du projet lui confereplusieursavantages comparatifs par rapport àd'autres centres touristiques de la région se trouvant en phase de planification et d'exécution. Parmi ces avantages, on dénombre, entre autres:

1) Sa proximité à l'aéroport international Playa de Oro à Manzanillo, à 28 kilomètres de distance ou 20 minutes de trajet, soit une distance optimale et concurrentielle pour attirer les flux touristiques.
2) Son interconnexion directe avec le système routier fédéral et celui de l'Etat, ainsi qu'avec les systèmes ferroviaire et maritime, lui a permis de s'intégrer aux principaux secteurs du marché touristique et aux principaux centres de production et de distribution de biens et services dans la région centrale et occidentale du pays.

3) Sa contiguité avec les localités dotées d'infrastructures de base et d'équipements, lui permet de recruter et de spécialiser la main-d'oeuvre localesans avoir besoin de créer ni d'élargir de nouveaux pôles urbains.

4) Sa localisation, presque à égale distance des centres touristiques de Manzanilloetde Puerto Vallarta, offre d'amplespossibilités pourl'intégration d'un développement touristique autonome, différent et sophistiqué dansla région, en même temps qu'elle lui permet de s'intégrer en termes compétitifs aux principaux centres touristiques sur le Pacifique que sont Acapulco et Ixtapa-Zihuatanejo dans l'Etat de Guerrero; Huatulco dans l'Etat de Oaxaca, Los Cabos et Mazatlán respectivementdanslesétats du sud de la Basse Californie et de Sinaloa.

5) Son emplacement stratégique, dans une zone prioritaire à vocation touristique, lui permet de contribuer et de bénéficier des efforts pour parfaire le corridor touristique Manzanillo-Barra de Navidad, qui implique l'union. économiqueet sociale de toutela côte, jusqu'à Barra de Navidad, avec Manzanillo.

\section{Vocation touristique de la zone}

Considérant la municipalité deManzanillo comme lieu du projer Isla Navidad, on peut souligner, entre autres, les facteurs suivants qui démontrent l'importance du tourisme pour le développement de cettezone:

- L'activité motrice du système économique est constitué par le tourisme et les services connexes.

- Le secteur touristique constitue une des principales sources de crétion $d^{*}$ emplois permanents et temporaires.

- Il existe plusieurs ressources touristiques naturelles, renouvelables et nonrenouvelables, qui n'ont toujours pas été exploitées à leur maximum et qui, par conséquent, constituent des éléments stratégiques pour consolider et développer de nouveaux projets.

- En raison de la croissance dynamique du tourisme dans la municipalité de Manzanillo, il s'est développée une expérience corporative dans les domaines administratif et hôtelier où l'on retrouve maintenant du personnel spécialisé.

- La région a réussi à s'implanter sur les marchés touristiques nationaux et internationaux.

- Elle dispose d'infrastructures de base (routes, chemins de fer, aéroport et port) et de services publics d'une capacité suffisante pour répondre à une augmentation du fluxdevisiteurs dans la région.

- Il cxiste une réserve territoriale suffisante pour assurer l'augmentation de l'offre hôtelière, tout au long de la côte dela municipalitéde Manzanillo.

Concept général du plan directeur de développement Isla Navidad

\section{Pbilosopbie de développement}

Un des traits les plus importants pour caractériser Isla Navidad, est celui de créer une communauté autonome dans laquelle le promoteur et les investisseurs immobiliers auront le contrôle administratif; c'est ainsi que la notion de propriété füt conçue et enregistrée comme Condominio Maestro Isla Navidad, sous le régime de CondominioHorizontal. Cerégimeinclut les constructions, les installations et autres 
accessoires érigés ou rattachés à l'immeuble.

Le processus d'exécution du méga-projet Isla Navidad comprend deux phases complémentaires de développement: la première consiste en la mise en place d'infrastructures, d'installations et d'équipements sur l'ensemble de la super ficie disponible; la deuxième se traduit par l'exécution de projets particuliers ou autonomes (hôtels, condominiums, habitations résidentielles et aires de services) sur les sites prévus pour la vente et pour des fins récréatives.

Dansle butdedévelopper un centre récréorésidentiel d'excellence, on a établi un ensemble de règles relatives à l'utilisation du sol, tels: une densité maximale de construction et une superficie maximale de déboisement. On cherche ainsi à mettre sur le marché un produit équilibré entre la rentabilité économique et financière et la préservation du milieu naturel. La construction d'ẻléments architectoniques distincts devra s'adapter au milieu naturel prédominant. Le maintien des traits du paysage du site confere à Isla Navidad un caractère unique. Le bosquet tropical original présentedes formesetdescouleurs dont l'enchantement et la beauté seront rehaussés pour maintenir ses caractéristiques naturelles.

Une attention particulière a été portée à la connaissance et à l'interprétation de l'environnement physico-naturel et socioéconomique, ainsi qu'auximpacts possibles qui pourraient se retrouver dans les composantes du milieu. La quantification (positive ou négative) des effets prévus sur le milieu, a permis d'établir une stratégie orientée vers le contrồle, la préservation et l'enrichissementdes systèmesécologiques.

\section{Phan directeur de latilisation du sol}

La superficie disponible pour développer le projet est divisée en deux types d'utilisation du sol clairement définis: le premier correspond à la superficie qui sera vendue et qui représente $91,2 \%$ de la totalité disponible alors que le second correspond à l'aire commune, soit $8,8 \%$ de la superficie restante.

À son tour, l'aire destinée à la vente est subdivisée en trois sections complémentaires: la section où seront construits les condominiums, hôtels, résidences et commerces; l'autre réservée pour le terrain de golf et les services connexes, et la dernière constituant la réserve de terrains.

La superficie touristico-résidentielle totalisant 276,9 hectares est subdivisée en 11 zones: Puerto Marino; El Palmar del Caracol; Centro Comercial del Lago; La Cascada; Vista Azul; Lomas del Sol; Pélicanos, El Faro, Punta de Oro; Campo de Golf et Villa Marina.

En considérant les investissements réalisés jusquà maintenant, on estime que la concrétisation de ce projet pourrait impliquer un investissement total de 1 milliard 505,6 millions de dollars.

\section{Stratégie générale de construction Isla Navidad}

Progranme d'installation des infrastructures et des équipements

Afin de réaliser leméga-projet Isla Navidad, des échéanciers de travail ont été établis spécifiantles activités à être réa lisées, autant dans les 11 zones de développement que dans celles récréatives et celles réservées.

\section{Programme d'exícution des projets moteur}

Pour appuyer le programme de commercialisation, de promotion et de publicité - et comme étape du processus de démarrage du projet et du début de la construction (1992-1994)- le groupe promoteur de l'Tsla Navidad a effectué d'importants investissements destinés à la réalisation de projets moteurs. La fin de la réalisation de ces projets est prévue pour le milieu de 1994.

\section{Programme de construction des équipenents} touristiques (1992-2005)

La construction etl'opération des différents équipements touristiques, qui feront partie du méga-projet Isla Navidad, sont prévues dans le cadre d'une stratégie d'installation à court, moyen et long termes.

Pour la mise en place et l'opération des différents équipements, trois périodes de développement complémentaires et subséquentes ont été prévues.

Dans un premier temps (1992-1995), lors du démarrage et du début de la construction, on prévoit développer approximativement $25 \%$ de l'offre touristique totale. Durant la deuxiè̀me étape (1996-2000), celle de croissance dynamique, on vise un développement de l'ordre de $45 \%$ de l'offre. Pour la troisième période (2001-2005), celle de consolidation et d'opération Isla Navidad dans sa totalité, on a prévu développer $30 \%$ restant de l'offre.

\section{Projets moteurs}

\section{Hôtel à Puerto Marino}

Parmi les projets moteurs à être développés dans l'Tle Navidad, signalons la construction et l'opération de l'hôtel Grand Bay Hotel Puerto de la Navidad à Puerto Marino.

Le complexe, d'une architecture raffinée, offrira en plus d'une série d'installations hôtelières, des chambres et des suites, des salles de bals et de congrès, des restaurants, des bars, terrasses, balcons, patios et fontaines, des arcades et jardins, ainsi qu'un SPA et un club de yacht.

\section{Le Mesón}

Faisant partie de l'hôtel Grand Bay Hotel Puerto de la Navidad à Puerto $M$ arino, cet équipement touristique de première classe aura, entre autres comme objectif, d'accueillir et d'assister tous les visiteurs qui désirent participer personnellement et investir dans quelques-uns des projets, tels que dans les ensembles de condominiums, de résidences et d'hôtels prévus dans le développement du projet. Cetéquipement sera ensuite converti en Centre de promotion et de ventes.

\section{Complexe résidentiel}

La construction d'ilots résidentiels a été prévue dans différentes zones.

Un premier îlot sera désigné sous le nom de The Stars Village ou le Peuple des Étoiles, et sera développé par l'un des professeurs de golf les plus connus au monde.

\section{Ensemble de condoniniums à curactère résidentiel}

Divers groupes de condominiums privés d'unequalité incomparable etintégrésdans le paysage de pâturages et de jardins ont été planifiés. Ils seront entourés par les verts du terrain degolfetrépartis dansl'ensemble du développement. 
Ces ensembles permettront de capter différents segments du marché national et international de haut niveau tels que ceux des golfeurs et des amateurs de tennis.

\section{Club de Yatch}

Afin d'intégrer pleinement l'opération des marinas (â Puerto Marinoetà Villa Marina) aux services touristiques, le développement Isla Navidad comprendra un club de Yatch haute gamme où les propriétaires des embarcations pourront devenir membre d'un club sélect.

\section{Rando Tenis}

Afin de développer ce nouveau concept, on a consacréunevastezone localisée aucentre du terrain de golf.

\section{Hôtel El Caracol}

On a également prévu la construction d'un hôtel qui sera enclavé dans la partie montagneuse de la zone des Palmas del Caracol, avec vue panoramique et services de Gran Turismo.

\section{Impact de l'Isla Navidad sur l'industrie touristique}

La stratégie de lotissement qui caractérisera Isla Navidad, se veut progressive et en conformité avec les projections établies quant au comportement del'offre hôtelière aux niveaux national et régional.

En accord avec ces projections on estime qu'en l'an 2005, lorsquele méga-projet Isla Navidad fonctionnera tel que prévu, il aura une incidence de $0,9 \%$ sur la disponibilité de chambres hôtelières au niveau national.

Cettecroissance aura lieu dans un contexte hautement compétitif, qui impliquera une augmentation des services actuels qu'offre I'hồtellerie de Gran Turismo et de 5 étoiles.

\section{Impacts socio-économiques}

Isla Navidad constituera un facteur d'importance primordiale dans la génération et la distribution de la richesse, non seulement dansl'économielocalemaiségalement dans l'économie régionale et nationale.

La construction desdifférents équipements touristiques et sportifs, encadrée dans une stratégie à court, moyen et long termes, pourrait générer 14,981 emplois directs et 37,453 indirects.

L'exécution du méga-projer Isla Navidad pourrait bénéficier à une population totale approximative de 139670 personnes.

La population totale qui en bénéficiera se divise ainsi: 1) les principaux centres de population à proximité et entourant Isla Navidad, tels: Barra de Navidad, Cihuatlán, Jaluco, San Patricio, La Huerta, Casimiro Castillo à Jalisco, et Manzanilloet Santiago à Colima. Ces populations, de par leur localisation, auront un impact fondamental sur le projet, non seulement dans l'approvisionnement d'aliments, de matières premières et de biens de production, mais aussi sur la disponibilité d'une maind'oeuvre qualifiếe et non-qualifiée; 2) les principaux centres de production d'aliments, debiens deproduction etdematières premières du reste du pays, particulièrement ceux des régions occidentale et centrale, deviendront de par leur proximité à l'Isla Navidad, la principale source d'approvisionnement pour les projets en opération.

L'industrie touristique est reconnue pour agir positivement sur l'utilisation de la main-d'oeuvre, l'apport de devises et sur la capitalisation d'autres secteurs; elle est considérée commeétant l'une des branches les plus importantes de l'économie, en raison de son rôle dans la répartition des salaites pour le bénéfice de la société.

\section{Conclusion}

Le méga-projet Isla Navidad, évalué rigoureusement par rapport à sa faisabilité urbanistique, économique et financière, s'est avéré́ concluant quant à sa viabilité, autant sous l'aspect urbain et économique que financier.

Ainsi, sur le plan urbain, parce qu'il constitue un équipement touristique nécessaire et prioritaire comme facteur de consolidation de la vocation touristique de la région Manzanillo-Puerto Vallarta.

Économiquement, parce que l'industrie touristique nationale se trouve dans une phase de récupération relative, suite à la récession sévère dont ont souffert l'activité et l'économie en général, et parce que le type d'offre projeté s'adresse stratégiquement aux secteurs du tourismenational et international à plus hauts revenus et à plus grande capacité de dépenses et d'apport de devises.

Le projet se justifie également par des avantages comparatifs par rapport à sa localisation, à l'exploitation des infrastructures portuaires, routières, et aéroportuaires existantes, à la disponibilité de la main-d'oeuvre dans les localités environnantes et à la structure de production dans les secteurs de l'agriculture, des pêcheries et de l'élevage dans la région.

Financièrement, parce que le potentiel d'investisseurs nationaux et étrangers assurera une demande de terrains pour fins immobilières dans le cadre d'une offre de haute qualité qui satisfait les attentes des touristes et des acheteurs. Il en résultera une demande plus dynamique qui rend possible la récupération del'investissement en un temps plus court, une réduction du risque général lié au profit et un degré de rentabilité plus intéressant.

Ainsi que la viabilité générale du projet est basée sur des conditions économico-financières qui caractérisent la conjoncture actuelle de l'économie nationale; elle se fonde également sur la santé de l'industrie touristique elle-même, pour laquelle une période de récupération et d'essor a été programmée par le Gouvernement fédéral, sur la demande prévisible des derniers investisseurs etacheteurs de condominiums et de résidences ainsi que sur la demande d'adhésion aux clubs nautiques, de tennis et de golf. $f$ 